\title{
Universality of the 1/3 Shot-Noise Suppression Factor in Nondegenerate Diffusive Conductors
}

\author{
T. González, C. González, J. Mateos, and D. Pardo \\ Departamento de Física Aplicada, Universidad de Salamanca, Plaza de la Merced s/n, E-37008 Salamanca, Spain \\ L. Reggiani \\ Istituto Nazionale di Fisica della Materia, Dipartimento di Scienza dei Materiali, Università di Lecce, \\ Via Arnesano, 73100 Lecce, Italy \\ O. M. Bulashenko and J. M. Rubí \\ Departament de Física Fonamental, Universitat de Barcelona, Avenida Diagonal 647, E-08028 Barcelona, Spain
} (Received 9 September 1997)

\begin{abstract}
Shot-noise suppression is investigated in nondegenerate diffusive conductors by means of an ensemble Monte Carlo simulator. The universal 1/3 suppression value is obtained when transport occurs under elastic collision regime provided the following conditions are satisfied: (i) The applied voltage is much larger than the thermal value; (ii) the length of the device is much greater than both the elastic mean free path and the Debye length. By fully suppressing carrier-number fluctuations, long-range Coulomb interaction is essential to obtain the $1 / 3$ value in the low-frequency limit. [S0031-9007(98)05732-9]
\end{abstract}

PACS numbers: 72.70. $+\mathrm{m}, 72.20 .-\mathrm{i}$, 73.23.Ad, 73.50.Td

In recent years kinetic phenomena in mesoscopic structures are offering a fascinating scenario for fundamental research [1]. One of the most up-to-date subjects is shotnoise suppression in disordered conductors. Here, the excess noise power has been predicted to comprise exactly one-third of the full shot-noise value $S_{I}=2 e I$. This result has been credited to different theoretical approaches as applied to several microscopic models of disordered conductors. For a phase-coherent model Beenakker and Büttiker [2] obtained the result using a bimodal distribution of transmission eigenvalues with the help of random matrix theory to calculate averages. For a semiclassical 1D model which includes Pauli principle Nagaev [3] found the same result using a Boltzmann kinetic approach within an elastic and energy independent relaxation-time approximation. For a semiclassical sequential tunneling model de Jong and Beenakker [4] obtained the 1/3 value within a Boltzmann-Langevin approach in the limit of an infinite number of equal barriers and independently from the value of their transmission coefficient. Compatible results have been found by Liu et al. [5] from a semiclassical implementation of a Monte Carlo simulation which includes Pauli principle. For a phase-coherent model Nazarov [6] has proven the universality of this result in the diffusive limit for arbitrary shape and resistivity distribution of the conductor as long as its length is greater than the carrier mean free path. Experimental evidence of the reduced shot-noise level close to the predicted $1 / 3$ value in diffusive mesoscopic conductors has been provided in [7-9].

From the above it is argued that the $1 / 3$ value of the suppression factor $\gamma=S_{I} / 2 e I$ is a universal phenomenon whose physical meaning should lay beyond classical or quantum mechanics and originate from some unifying concept. The aim of this Letter is to address this issue. We conjecture that discreteness of charge transport is at the basis of such a concept, and that a transport dominated by elastic interactions is ultimately the physical reason for the $1 / 3$ suppression independently from the quantum or classical approach used. Both the (apparently unrelated) coherent [2] and semiclassical [3] contexts where the reduction factor $1 / 3$ has appeared assume a degenerate Fermi gas, and the noise reduction comes from the regulation of electron motion by the exclusion principle. Landauer claims that the appearance of $1 / 3$ in these very different cases is a numerical coincidence [1]. We argue that neither phase coherence nor Fermi statistics are required for the occurrence of suppressed shot noise in diffusive conductors. We show a third case where the origin of the effect is completely classical, and the correlation between electrons comes from their Coulomb interaction, rather than the exclusion principle. The repeated occurrence of $1 / 3$ leads us to believe that this must be more than a numerical coincidence.

To support the above conjecture, we present the results of a Monte Carlo simulation for a nondegenerate diffusive conductor. By providing an exact solution of the kinetic equation coupled with a Poisson solver (PS) we are free from any approximation and thus in the position to obtain a rigorous proof of our findings. Furthermore, the role of long-range Coulomb interaction [10,11], although known since the times of vacuum diodes [12], is here considered for the relevant case of a medium in the presence of elastic and inelastic scattering for the first time.

For the calculations we consider the following simple model: a lightly doped active region of a semiconductor sample of length $L$ sandwiched between two heavily doped contacts injecting carriers into the active region. 
The contacts are considered to be Ohmic (the voltage drop inside them being negligible) and to remain always at thermal equilibrium. Thus, electrons are emitted from the contacts according to a thermal-equilibrium MaxwellBoltzmann distribution at the lattice temperature $T$, and they move inside the active region following the classical equations of motion by undergoing isotropic scattering in momentum space. To exclude additional correlations due to Fermi statistics, the electron gas is assumed to be nondegenerate. In principle, electrons are injected with a Poissonian statistics, i.e., the time between two consecutive electron emissions is generated with a probability $P(t)=\Gamma e^{-\Gamma t}$, where $\Gamma=\frac{1}{2} n_{c} v_{\mathrm{th}} S$ is the injection-rate density, $v_{\text {th }}=\sqrt{2 k_{B} T / \pi m}$ the thermal velocity, $S$ the cross-sectional area of the device, and $m$ the electron effective mass. However, to prove our conjecture that the origin of $\gamma=1 / 3$ is just elastic scattering and therefore independent of the contact injection, the fluctuating emission rate at the contacts in the diffusive limit is taken to follow other kind of statistics ranging from uniform to Poissonian. For the simulations we have used the following set of parameters: $T=300 \mathrm{~K}, m=0.25 m_{0}\left(m_{0}\right.$ being the free electron mass), relative dielectric constant $\varepsilon=11.7, L=200 \mathrm{~nm}$, and $n_{c}=4 \times 10^{17} \mathrm{~cm}^{-3}$ (much higher than the sample doping). The above set of values yields for the dimensionless parameter $\lambda=L / L_{D c}$ (with $L_{D c}$ the Debye length corresponding to $n_{c}$ ), which characterizes the importance of the electrostatic screening [10], the value $\lambda=30.9$, which implies significant space-charge effects and inhomogeneity inside the structure. Furthermore, the average time between collisions in the bulk $\tau$ is assumed to be independent of energy, and is varied from $10^{-15} \mathrm{~s}$ to $10^{-11} \mathrm{~s}$, so that both regimes of carrier transport, ballistic $(\ell / L \gg 1)$ and diffusive $(\ell / L \ll 1)$, are covered. In our calculations $\ell$, the carrier mean free path, is estimated as $v_{\text {th }} \tau$. To analyze the effect of scattering inelasticity, collisions are treated as elastic or as inelastic. In the latter case the carrier is thermalized after each scattering event.

We apply a $d c$ voltage and calculate the time-averaged current $I$ and the current autocorrelation function $C_{I}(t)$ by means of an ensemble Monte Carlo simulator selfconsistently coupled with a PS. We assume that the active region of the structure in transversal directions is sufficiently thick to allow a 1D electrostatic treatment. Accordingly, the simulation is $1 \mathrm{D}$ in real space and $3 \mathrm{D}$ in momentum space. We stress that in our approach the number of electrons $N$ inside the sample fluctuates in time due to the random injection/extraction from the contacts, and we can evaluate both the time-averaged value $\langle N\rangle$ and its fluctuations. To analyze the importance of the effects associated with long-range Coulomb interaction, we provide the results for two different simulation schemes [10]. The first one involves a dynamic PS, where the potential is self-consistently updated at each time step during the simulation by solving the Poisson equation under the condition that the contact potential remains time indepen- dent. The second scheme makes use of a static PS, so that only the stationary potential profile is calculated and carriers move in the frozen nonfluctuating electric field profile. Both schemes give exactly the same average current and steady-state spatial distributions of all the quantities, but the noise characteristics are in general quite different.

Figure 1 presents the results for the low-frequency suppression factor $\gamma=S_{I} / 2 e I$ as a function of $\ell / L$ for an applied voltage of $U=40 k_{B} T / e$. When the transport is ballistic or quasiballistic $\left(\ell / L \geqslant 10^{-1}\right)$, the static results show full shot noise $(\gamma \approx 1$ within numerical uncertainty) associated with the carrier injection (which in this range is modeled as Poissonian), whereas in the case of the dynamic results space charge is responsible for a relevant noise suppression, yielding for the suppression factor the value of 0.045 under perfect ballistic regime $(\ell / L \gg 1)[10]$. The observed reduced shot-noise level is accompanied by a sub-Poissonian electron number statistics [11]. In the transition from ballistic to diffusive regime, the suppression due to long-range Coulomb interaction remains active, being more pronounced in the case of inelastic scattering mechanisms. Under fully diffusive regime, attained at $\ell / L \lesssim 10^{-2}$, the results become independent of the carrier injecting statistics and the following asymptotic conditions are detected. The static results, both in the elastic and inelastic cases, keep the full shot-noise limit. The elastic-dynamic case attains the $1 / 3$ value within numerical uncertainty in excellent agreement with theoretical expectations. The inelasticdynamic case is further suppressed well below $1 / 3$.

Figure 2 complements the results of the elastic dynamic case in Fig. 1 by presenting calculations at increasing applied voltages. Here we find the remarkable fact that, for high voltages, the $1 / 3$ value in the diffusive limit is reached from the full shot-noise value in the ballistic limit. The reason for the different behavior in the ballistic regime is the presence or absence of a potential barrier

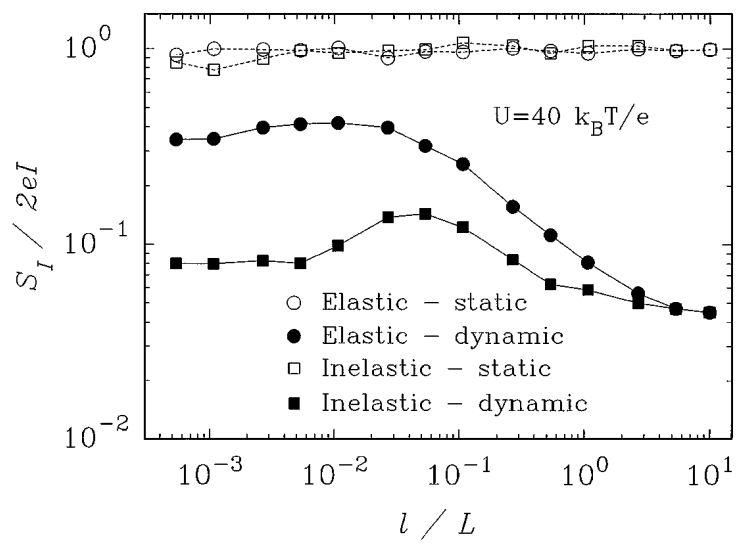

FIG. 1. Shot-noise suppression factor vs ballistic parameter $\ell / L$ for an applied voltage of $U=40 k_{B} T / e$ calculated by using static (open symbols, dashed line) and dynamic (full symbols, solid line) potentials and elastic (circles) and inelastic (squares) scattering mechanisms. 


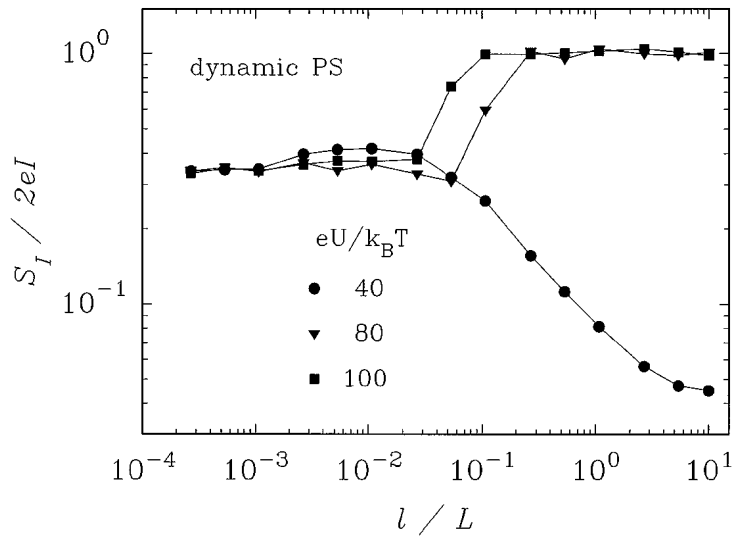

FIG. 2. Shot-noise suppression factor vs ballistic parameter $\ell / L$ for the case of elastic scattering mechanisms at different applied voltages. Calculations are performed by using dynamic (self-consistent) potential.

near the cathode which controls the current through the structure. For the highest voltages, when the barrier disappears, the current saturates and the suppression factor takes on the full shot-noise level as for the static case [10]. It has been checked that the onset of the $1 / 3$ value takes place when, because of intensive elastic scattering, a significant energy redistribution among the three velocity components is achieved. Accordingly, the higher the applied voltage, the larger the energy gained by electrons in the free flights and, consequently, more scattering is needed to redistribute the energy equally among the components. Hence, the threshold of the $1 / 3$ regime is shifted towards lower values of $\ell / L$ as the applied voltage increases. We notice that the $1 / 3$ limit exhibits several universal properties, namely, it is independent of (i) the applied voltage bias (see Fig. 2), (ii) the scattering strength, (iii) and the carrier injecting statistics.

To illustrate and explain the physical origin of the $1 / 3$ value, Fig. 3 reports a typical frequency spectrum of the suppression factor $S_{I}(f) / 2 e I$ under elastic-diffusive conditions for static and dynamic PS. Monte Carlo simulation allows the calculation of the three terms in which $S_{I}(f)$ can be decomposed, namely velocity, number, and cross-correlation contributions $S_{I}(f)=S_{V}(f)+$ $S_{N}(f)+S_{V N}(f)[10]$. In the static case the spectrum clearly shows that all three terms contribute to $S_{I}(f)$, and two different time scales can be identified. The longest one, associated with the transit time of the carriers through the device $\tau_{T}$, is evidenced in the terms $S_{N}$ and $S_{V N}$. The shortest one, related to the relaxation time of elastic scattering $\tau$, is manifested in $S_{V}$. The latter is responsible for $1 / 3$ (within the numerical uncertainty) of the full shot-noise value obtained for the suppression factor at low frequency, while the former provides the rest of the contribution up to the value 1 . In contrast with this behavior, in the dynamic case the number contribution is found to be compensated by a negative velocity-number cross-correlation contribution and, as

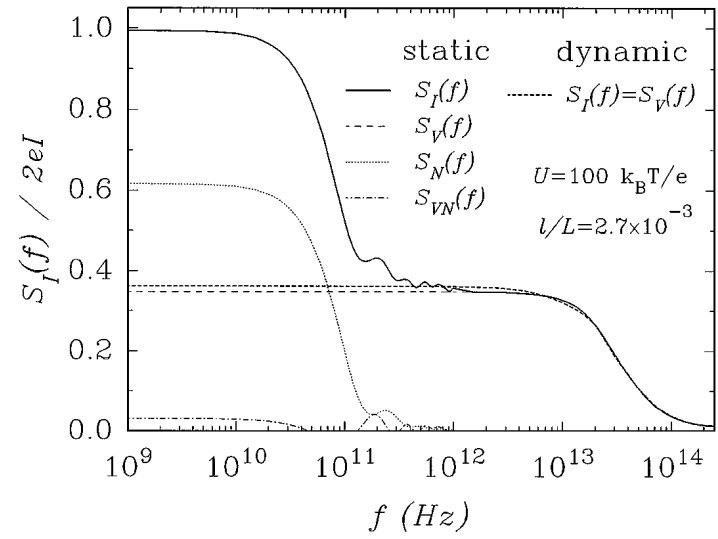

FIG. 3. Spectrum of the shot-noise suppression factor for the case of static and dynamic potentials under elastic-diffusive conditions. Different contributions to the total value are indicated in the figure.

a result, $S_{I}(f)$ is found to coincide with $S_{V}(f)$ in all the frequency range, thus showing only the cutoff at higher frequencies. It is important to notice that in the frequency range $\frac{1}{2 \pi \tau_{T}} \lesssim f \lesssim \frac{1}{2 \pi \tau}$ both static and dynamic suppression factors exhibit the $1 / 3$ value, which is related to velocity fluctuations. However, at low frequency only the dynamic case takes this value by virtue of Coulomb correlations, which are responsible for the mutual compensation of $S_{N}$ and $S_{V N}$ contributions. For this compensation to take place we have checked that it is necessary to fulfill the condition $L \gg L_{D c}$ in order to achieve a significant action of long-range Coulomb interaction.

Figure 4 reports the low-frequency suppression factor in the diffusive regime $\left(\ell / L=10^{-3}\right)$ as a function of the applied voltage, calculated by using the dynamic PS. We stress that these results are independent of the injecting statistics at the contacts. By comparing the elastic and inelastic cases we find that at the lowest bias both

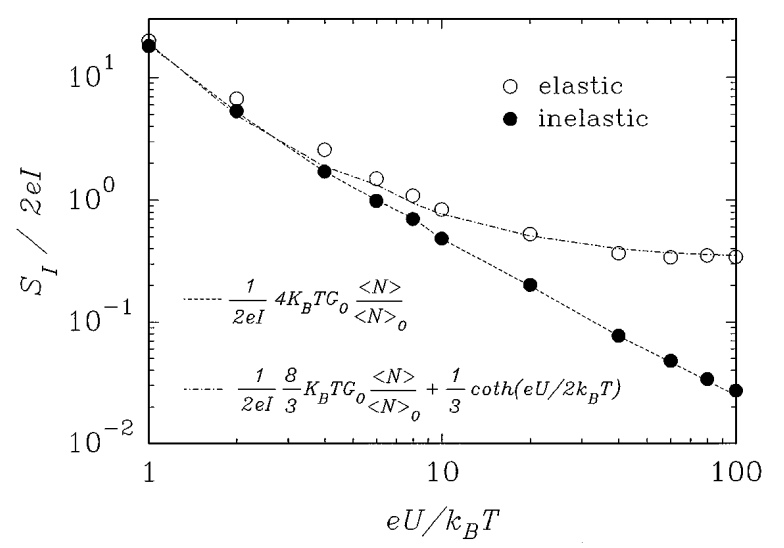

FIG. 4. Shot-noise suppression factor vs applied bias $U$ calculated with dynamic potential for the cases of elastic and inelastic scattering mechanisms with $\ell / L=10^{-3}$ and an injecting concentration $n_{c}=4 \times 10^{17} \mathrm{~cm}^{-3}$. The curves correspond to the fittings of Eqs. (1) and (2). 
cases coincide by providing the standard thermal noise as predicted by Nyquist relation. On the contrary, at the highest voltages the elastic case achieves the $1 / 3$ limiting value, in full agreement with our conjecture, while the inelastic case continues decreasing.

The results for the low-frequency value of the spectral density in the inelastic case $S_{I}^{\text {inel }}$ are closely fitted by the simple relation:

$$
S_{I}^{\text {inel }}=4 k_{B} T G_{0} \frac{\langle N\rangle}{\langle N\rangle_{0}},
$$

where $G_{0}$ is the conductance and $\langle N\rangle_{0}$ the average number of electrons inside the sample, both in the limit of vanishing bias. This result means that, under strong inelastic scattering, the noise in our sample is just the thermal Nyquist noise (modulated by the variation in the number of carriers) even in the presence of a high bias and a net current flowing through the sample. Therefore, inelastic scattering strongly suppresses shot noise and makes the noise become macroscopic $(\gamma \ll 1$; see Fig. 4). These results confirm previous predictions by Liu and Yamamoto [13] and Nagaev [14], with the important difference that our approach is completely classical. The conclusion of Shimizu and Ueda [15] that, while dephasing is irrelevant, energy transfer from electrons to other systems is essential for shot-noise suppression, is also supported by the present results.

The values of the low-frequency spectral density in the elastic case $S_{I}^{\mathrm{el}}$ are nicely reproduced by the expression:

$$
S_{I}^{\mathrm{el}}=\frac{8}{3} k_{B} T G_{0} \frac{\langle N\rangle}{\langle N\rangle_{0}}+\frac{2}{3} e I \operatorname{coth}\left(\frac{e U}{2 k_{B} T}\right),
$$

which is quite similar to that obtained by Nagaev [3], and describes the crossover from thermal-Nyquist noise for $e U \ll k_{B} T\left(S_{I}^{\mathrm{el}}=4 k_{B} T G_{0}\right)$ to suppressed shot noise for $e U \gg k_{B} T\left(S_{I}^{\mathrm{el}}=\frac{2}{3} e I\right)$, thus providing $\gamma=1 / 3$ for the highest applied voltages (Fig. 4). The main difference between the effect of elastic and inelastic scattering on the noise in our model is the following: Strong inelastic scattering, by dissipating the energy that electrons gain from the field and randomizing the momentum, reduces the electron average energy to that of the lattice, and therefore the noise corresponds to thermal-Nyquist noise at any bias. On the other hand, elastic scattering, by simply randomizing the electron momentum, allows for the broadening of the electron velocity distribution at high voltages. As a consequence, the level of noise increases with respect to equilibrium conditions, which results in a noise power with value $1 / 3$ of the full shot-noise value [16]. In addition, we have checked that if a 2D momentum space is considered (electron energy is shared between two velocity components after each scattering), the observed $\gamma=S_{I}^{\mathrm{el}} / 2 q I$ in diffusive regime is about $1 / 2$, which indicates that the suppression factor is related to the momentum-space dimensionality [17].

In conclusion, within an ensemble Monte Carlo scheme we have investigated the shot-noise suppression in non- degenerate diffusive conductors. Results prove that the $1 / 3$ value of the suppression factor is related to a transport dominated by elastic scattering (diffusive limit) under the condition $e U \gg k_{B} T$. The appearance of this factor requires the simultaneous validity of the two conditions $\ell \ll L$ and $L_{D c} \ll L$, the former implying the achievement of fully diffusive conditions, the latter the decisive role of long-range Coulomb correlations in suppressing the contribution associated with number fluctuations. Inelastic scattering is found to strongly suppress shot noise, reducing it to thermal Nyquist noise under heavily dissipative conditions.

This work has been partially supported by the Comisión Interministerial de Ciencia y Tecnología through Project No. TIC95-0652 and Ministero dell' Università e della Ricerca Scientifica e Tecnologica (MURST). O.M. B. acknowledges support by the Dirección General de Enseñanza Superior, Spain.

[1] R. Landauer, Physica (Amsterdam) 227B, 156 (1996).

[2] C. W. J. Beenakker and M. Büttiker, Phys. Rev. B 46, 1889 (1992).

[3] K. E. Nagaev, Phys. Lett. A 169, 103 (1992).

[4] M. J. M. de Jong and C. W. J. Beenakker, Phys. Rev. B 51, 16867 (1995).

[5] R. Liu, P. Eastman, and Y. Yamamoto, Solid State Commun. 102, 785 (1997).

[6] Yu. V. Nazarov, Phys. Rev. Lett. 73, 134 (1994).

[7] F. Liefrink, J. I. Dijkhuis, M. J. M. de Jong, L. W. Molenkamp, and H. van Houten, Phys. Rev. B 49, 14066 (1994).

[8] A. H. Steinbach, J. M. Martinis, and M.H. Devoret, Phys. Rev. Lett. 76, 3806 (1996).

[9] R. J. Schoelkopf, P. J. Burke, A. A. Kozhevnikov, D.E. Prober, and M. J. Rooks, Phys. Rev. Lett. 78, 3370 (1997).

[10] T. González, O. M. Bulashenko, J. Mateos, D. Pardo, and L. Reggiani, Phys. Rev. B 56, 6424 (1997).

[11] O. M. Bulashenko, J. Mateos, D. Pardo, T. González, L. Reggiani, and J. M. Rubí, Phys. Rev. B 57, 1366 (1998).

[12] A. J. Rank, Bell. Syst. Tech. J. 17, 592 (1938); B. J. Thompson, D. O. North, and W. A. Harris, RCA Rev. 4, 269 (1940); 4, 441 (1940).

[13] R. C. Liu and Y. Yamamoto, Phys. Rev. B 50, 17411 (1994).

[14] K. E. Nagaev, Phys. Rev. B 52, 4740 (1995).

[15] A. Shimizu and M. Ueda, Phys. Rev. Lett. 69, 1403 (1992).

[16] The relation of electronic noise with the breadth of the velocity distribution was already invoked in R. Landauer, Phys. Rev. B 47, 16427 (1993) and Ref. [14] in different contexts.

[17] The appearance of the $1 / 3$ factor in the $1 D$ simulation of Ref. [5], which is in apparent contradiction with this conclusion, is due to the effect of the Pauli exclusion principle, while in our case the correlation between electrons comes from their Coulomb interaction. 